\title{
HALF-TURN ROTATION OF A POLARITY INVERSION LINE AND ASSOCIATED QUADRUPOLAR-LIKE STRUCTURE IN THE SUN
}

\author{
Tetsuya Magara ${ }^{1}$, Jun-Mo An ${ }^{2}$, Hwanhee LeE $^{2}$, and Jihye Kang ${ }^{2}$ \\ ${ }^{1}$ Department of Astronomy and Space Science, School of Space Research, Kyung Hee University, Yongin, \\ Gyeonggi-do 446-701, Korea \\ E-mail : magara@khu.ac.kr \\ 2 School of Space Research, Kyung Hee University, Yongin, Gyeonggi-do 446-701, Korea \\ E-mail : ajmyaa@khu.ac.kr, lhhee@khu.ac.kr andsiriustar@khu.ac.kr \\ (Received June 8, 2011; Revised August 5, 2011; Accepted August 5, 2011)
}

\begin{abstract}
This paper reports a characteristic motion of a polarity inversion line (PIL) formed at the solar surface, which is newly found by performing a three-dimensional magnetohydrodynamic simulation of flux emergence in the Sun. A magnetic flux tube composed of twisted field lines is assumed to emerge below the surface, forming a bipolar region with a PIL at the surface. A key finding is the successive half-turn rotation of the PIL, leading to the formation of a quadrupolar-like region at the surface and a magnetic configuration in the corona; this configuration is reminiscent of, but essentially different from the so-called inverse-polarity configuration of a filament magnetic field. We discuss a physical mechanism for producing the half-turn rotation of a PIL, which gives new insights into the magnetic structure formed via flux emergence. This presents a reasonable explanation of the configuration of a filament magnetic field suggested by observations.
\end{abstract}

Key words : Sun: magnetic topology — Sun: surface magnetism - magnetohydrodynamics methods: numerical

\section{INTRODUCTION}

A polarity inversion line (hereafter PIL) is observed at the solar surface where positive and negative magnetic polarities coexist; it is defined as the boundary between these positive and negative polarity regions. A PIL constitutes the photospheric part of a magnetic structure extending from the solar interior to the atmosphere, inside which various objects are formed. For example, solar filament is one of these objects, which is observed as a long and thin body floating in the corona above a PIL. It is now well known that the magnetic field plays an important role in supporting a filament against gravity (Tandberg-Hanssen 1995), and hence the magnetic structure of a filament is the key in understanding the nature of a filament which sometimes shows a global eruption (Low 1996).

The detailed configuration of the magnetic field in the corona is usually hard to observe directly, although it can be inferred from the surface distribution of the magnetic field which is well observed. Regarding the magnetic field forming a filament, two major models have been proposed to explain the configuration of coronal magnetic field: the normal-polarity configuration (Kippenhahn \& Schluter 1957) and the inversepolarity configuration (Kuperus \& Raadu 1974), as illustrated in Fig. 1. Both models basically give the

Corresponding Author: T. Magara same surface magnetic-field distribution; however, they show quite different configurations in the corona. To clarify which model (or even a different one) reproduces the configuration of the filament magnetic field, we should focus on the formation process leading to the configuration suggested by these models. The formation process involves the evolution of the surface magnetic field and of a PIL.

A way of forming a magnetic structure is given by the emergence of a magnetic flux tube below the surface (see the right panel in Fig. 1). This is a direct consequence of the magnetic buoyancy working in the layers where the magnetized plasma is stratified under gravity such as the solar convection zone (Parker 1955). If a flux tube is composed of twisted field lines, which is suitable for keeping the integrity of a flux tube when it travels across the convection zone (Fan 2009), a bipolar region with a PIL is formed at the surface when the flux tube emerges. As the emergence further proceeds, the bipolar region becomes elongated along the PIL where local peaks of positive and negative magnetic polarities show a shear motion. A number of numerical simulations have successfully demonstrated these processes (Fan 2001; Magara \& Longcope 2003; Abbett \& Fisher 2003; Manchester et al. 2004; Archontis et al. 2004; Nozawa 2005; Murray et al. 2006).

On the other hand, observations indicate that a more complicated magnetic-field distribution exists at the surface than a simple bipolar distribution. Martin 


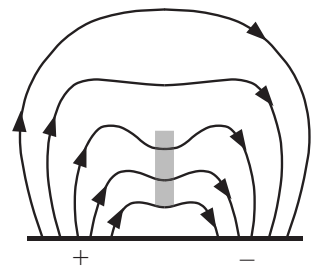

Normal-polarity

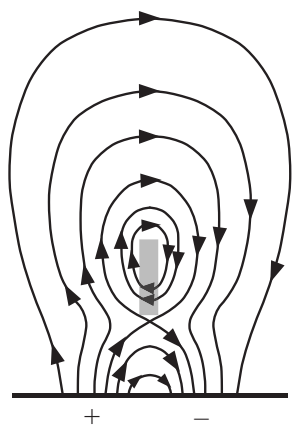

Inverse-polarity

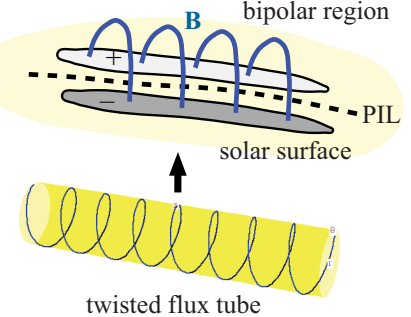

twisted flux tube

Fig. 1. - Models of a filament magnetic field (normal-polarity in the left panel and inverse-polarity in the middle panel). Adapted from Anzer (1987). The shaded area represents a filament. The right panel shows a scenario of an emerging flux tube, which forms a bipolar region with a PIL at the surface.

(1998) and Chae et al. (2001) show that there are small weak flux regions around a PIL, which have the opposite polarity with respect to the nearby major polarities composing a bipolar region. This suggests that the emergence scenario of a twisted flux tube mentioned above might be wrong, or that there is a missing process within this scenario which gives complexity to the surface distribution of the magnetic field.

This paper reports a new result on such a missing process, showing that a PIL presents a characteristic motion that causes a significant change in the surface distribution of the emerging magnetic field. A key finding is the half-turn rotation of a PIL, which changes the surface distribution from a simple bipolar distribution into a quadrupolar-like one, in agreement with the observations. In what follows, we show how the halfturn rotation of a PIL proceeds, and why this happens. We also discuss a possible configuration of the filament magnetic field obtained from the present study, by comparing it with the normal and inverse-polarity configurations.

\section{MODEL DESCRIPTION}

We performed a three-dimensional magnetohydrodynamic (MHD) simulation in Cartesian coordinates with the $z$-axis directed upward. A standard set of MHD equations may be written in the following way:

$$
\begin{gathered}
\frac{\partial \rho}{\partial t}+\nabla \cdot(\rho \mathbf{v})=0 \\
\rho\left[\frac{\partial \mathbf{v}}{\partial t}+(\mathbf{v} \cdot \nabla) \mathbf{v}\right]=-\nabla P+\frac{1}{4 \pi}(\nabla \times \mathbf{B}) \times \mathbf{B}-\rho g_{\mathrm{o}} \hat{\mathbf{z}} \\
\frac{\partial \mathbf{B}}{\partial t}=\nabla \times(\mathbf{v} \times \mathbf{B}) \\
\frac{\partial P}{\partial t}+\nabla \cdot(P \mathbf{v})=-(\gamma-1) P \nabla \cdot \mathbf{v}
\end{gathered}
$$

and

$$
P=\frac{\rho \Re T}{\mu},
$$

where $\rho, \mathbf{v}, \mathbf{B}, P, g_{0}, \gamma, \mu, \Re$, and $T$ indicate the gas density, flow velocity, magnetic field, gas pressure, gravitational acceleration, adiabatic index $(\gamma=5 / 3$ is assumed), mean molecular weight ( $\mu=0.5$ is assumed), gas constant, and temperature, respectively. We neglected partial ionization and such physical diffusive effects as thermal conduction and magnetic diffusion.

The initial state is in mechanical equilibrium, with a prescribed temperature. We assumed a simplified profile of temperature which depends only on $z$, based on a standard model of the solar atmosphere such as the VAL model (Vernazza et al. 1981). We used this profile to construct a hydrostatic atmosphere stratified under uniform gravity. The initial distributions of gas density (dashed line), pressure (dotted line), and temperature (dot-dashed line) of the plasma along the $z$-axis are presented in a graph inserted into Fig. 2.

A magnetic flux tube composed of twisted field lines started to rise from the sub-surface layer $(z<0)$ that is weakly unstable for convection motions. Fig. 2 shows a snapshot of the magnetic field lines taken at a developed phase of the simulation, in which the magnetic field partially emerges and expands to form a structure above the surface. The blue field lines are originally distributed in the vicinity of the axis of the flux tube (inner field lines), while the red field lines are in the outer boundary part of the flux tube (outer field lines).

The magnetic field initially assumes a straight flux tube with a finite radius (1 Mm), placed along the $y$ axis. The flux tube is composed of uniformly twisted field lines with a left-handed twist, which satisfies a force-free condition inside the tube (Gold \& Hoyle 1960) while keeping pressure balance at the boundary between the tube and the external field-free plasma. The temperature inside the flux tube is slightly reduced so that a hydrostatic condition is satisfied there. The initial depth of the tube axis is $2.6 \mathrm{Mm}$ below the sur- 


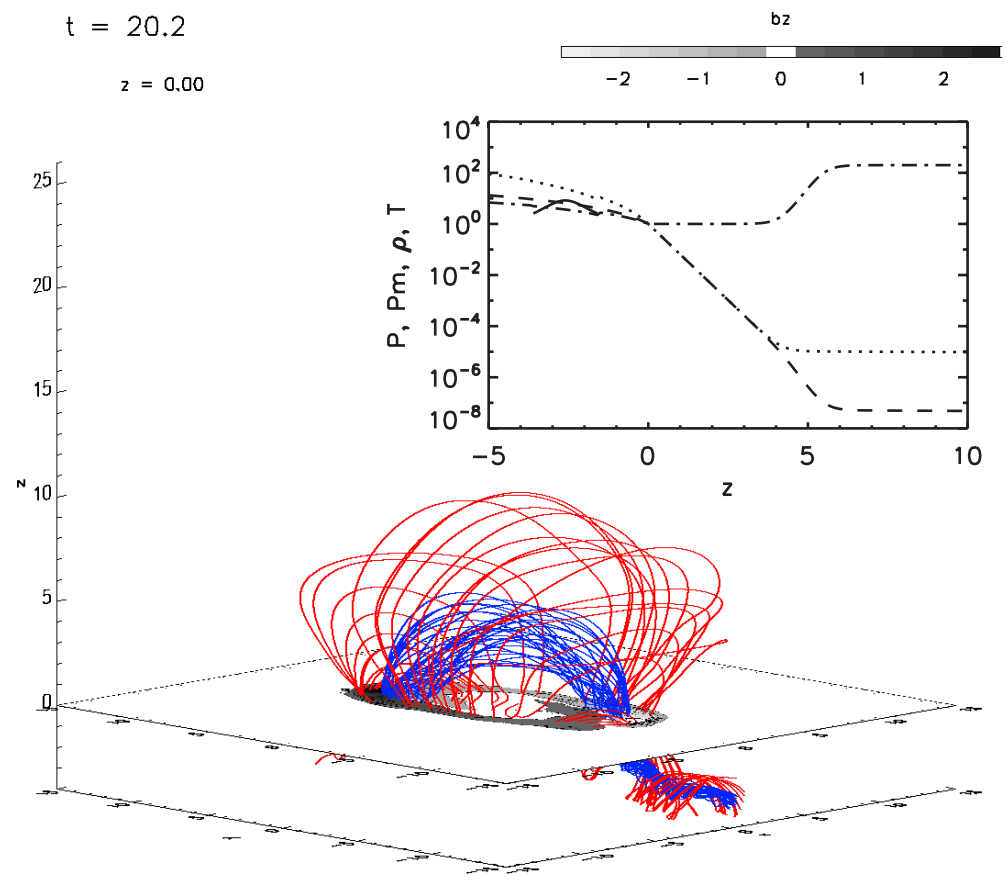

Fig. 2. - Snapshot of an emerging flux tube taken at a late phase of the simulation. The blue and red lines represent the field lines which are distributed initially near the axis and the boundary of the flux tube, respectively. The horizontal plane located at $z=0$ indicates the surface of the Sun, and a grey scale map on this plane shows the distribution of the vertical magnetic flux. The inset graph shows the initial distribution of physical quantities along the $z$-axis in logarithmic scale $(-5<z<10$ is presented). These quantities are gas pressure $(P$, dotted line), density $(\rho$, dashed line), temperature ( $T$, dot-dashed line), and magnetic pressure ( $P m$, solid line), scaled to their photospheric values (the magnetic pressure is scaled to the photopsheric value of the gas pressure). The length unit is in Mm. An animation of this emerging flux tube is available online (http://163.180.179.74/ magara/Download/EFT.mp4).

face, and the field lines make one helical turn around the axis while running $4.2 \mathrm{Mm}$ in the axial direction. The field strength takes a maximum of $6500 \mathrm{G}$ at the axis, and the total magnetic flux contained in the flux tube is about $10^{20} \mathrm{Mx}$. The value of the pre-emerged field strength has been carefully chosen so as to match the vertical magnetic flux observed at the surface, typically taking a few thousand Gauss in the main bipolar region (Okamoto et al. 2008). The initial distribution of the magnetic pressure along the $z$-axis is also presented in the inset graph (solid line). The units of length, velocity and magnetic field used in this simulation are given by $1 \mathrm{Mm}, 12.8 \mathrm{~km} / \mathrm{s}$, and $580 \mathrm{G}$, respectively.

The simulation started with the slow rise of the middle part of the flux tube, which was driven by imposing a sinusoidal velocity perturbation on the flux tube within $r \leq r_{f}$ and $-y_{0} \leq y \leq y_{0}$ for a short time $0 \leq t \leq t_{0}$

$$
v_{z}=v_{0} \frac{[1+\cos (2 \pi y / \lambda)]}{2} \sin \left(\frac{\pi}{2 t_{0}} t\right),
$$

where $r_{f}=1 \mathrm{Mm}, y_{0}=8 \mathrm{Mm}, t_{0}=39 \mathrm{~s}, v_{0}=3.8$ $\mathrm{km} \mathrm{s}^{-1}, \lambda=16 \mathrm{Mm}$. The subsequent temporal development was obtained by solving the MHD equations with a modified Lax-Wendroff method (Magara
1998). The total simulation domain is $(-40,-45,-8)$ $\mathrm{Mm} \leq(x, y, z) \leq(40,45,71) \mathrm{Mm}$. The grid spacing increases from $(\Delta x, \Delta y, \Delta z)=(0.1,0.2,0.1) \mathrm{Mm}$ towards $(1,1,2) \mathrm{Mm}$, giving the total grid number $N_{x} \times N_{y} \times N_{z}=233 \times 247 \times 256$. The finest grid occupies the region of $(-5,-15,-8) \mathrm{Mm} \leq(x, y, z) \leq(5,15,10)$ $\mathrm{Mm}$. We imposed a rigid boundary condition on the bottom boundary, whereas a free boundary condition was imposed on the other boundaries and a wavedamping zone was placed near all the boundaries.

\section{RESULTS}

Fig. 3 shows the temporal development of the magnetic field at the surface (left panels), where the grey scale map represents the vertical magnetic flux while the arrows indicate the horizontal magnetic field. An animation available at http://163.180.179.74/ magara /Download/EFT.mp4 shows the temporal development of the red and blue field lines presented in Fig. 2. The right panels in Fig. 3 show the temporal development of the emergence velocity $v_{e m g}$ introduced in Magara (2011a), which represents the net emergence $\left(v_{\text {emg }}>0\right)$ or sub-mergence $\left(v_{\text {emg }}<0\right)$ of the magnetic field at the surface. The relation between the emergence velocity and the vertical component of the 

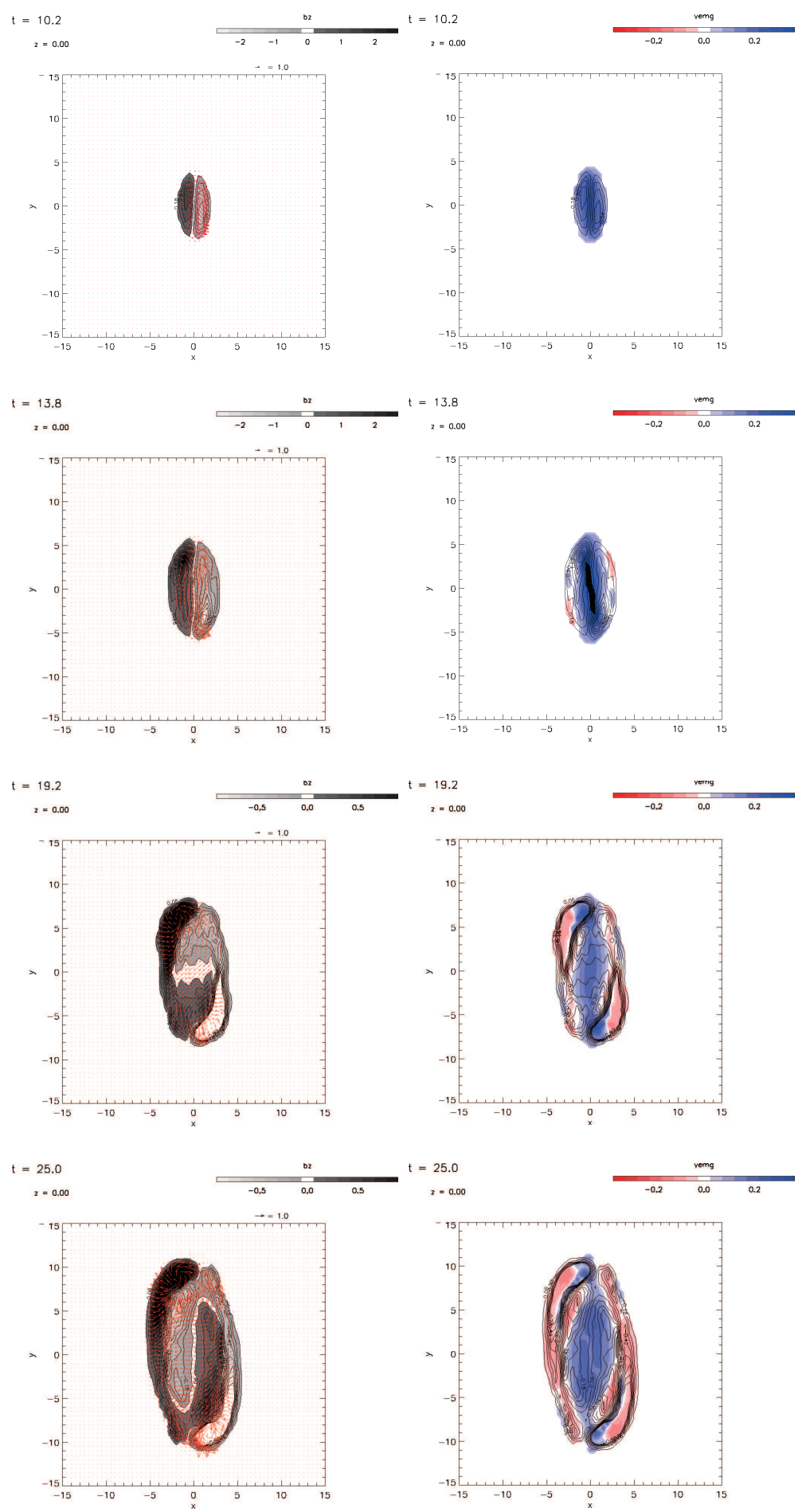

Fig. 3. - Temporal development of the surface distribution of the magnetic field (left panels), and emergence velocity (right panels) defined in Eq. (8). Grey scale maps with contours in the left panels show the vertical magnetic flux, while red arrows represent the horizontal magnetic field. Color maps and contours in the right panels represent the emergence velocity and the vertical magnetic flux, respectively.

Poynting flux is given by:

$$
F_{M z}=\iint_{z=0} \frac{B^{2}}{4 \pi} v_{e m g} d x d y
$$




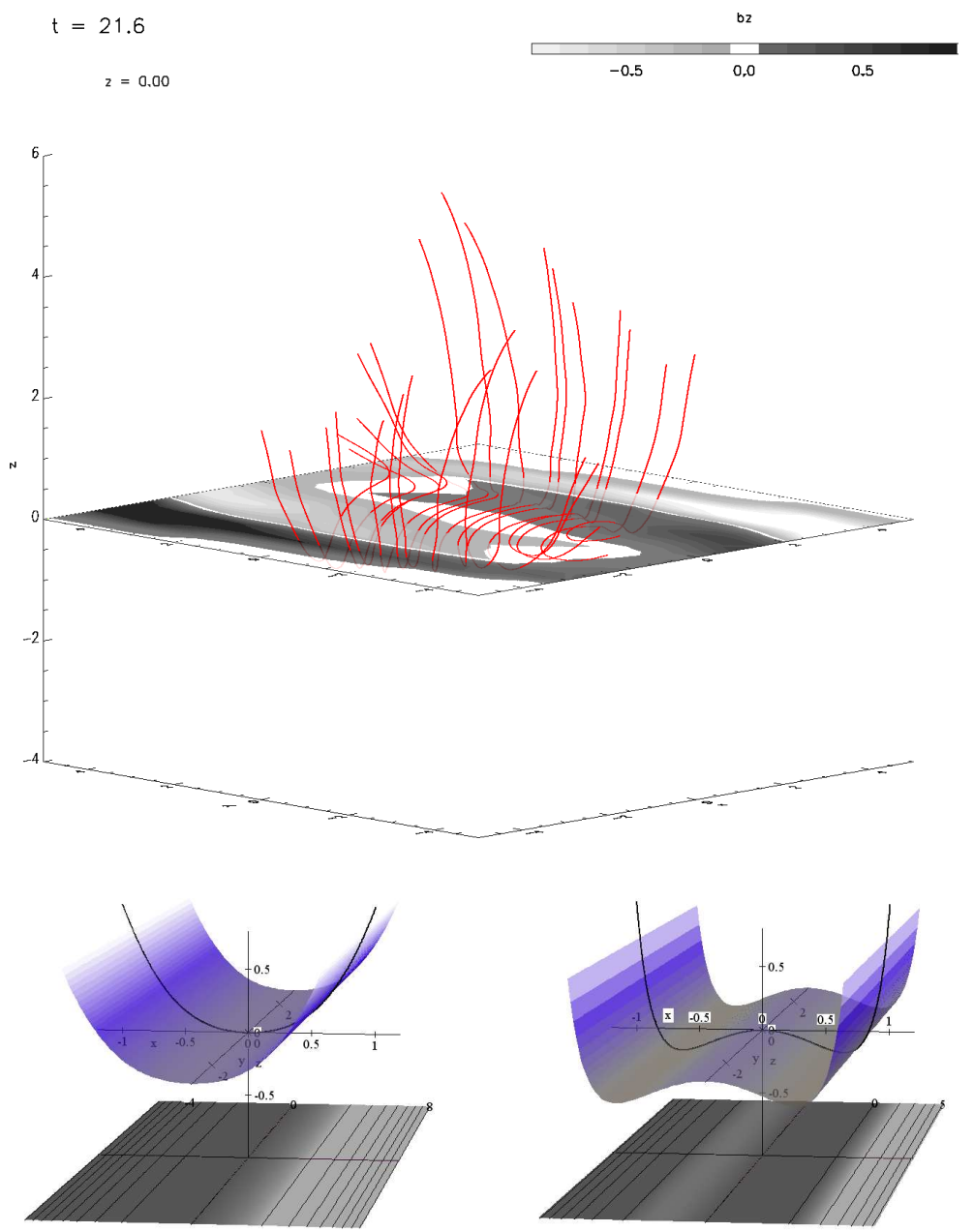

Fig. 4. - Top: Close-up view of a quadrupolar-like region at the surface. The grey scale map represents the surface distribution of the vertical magnetic flux while the magnetic field lines are given in red. Bottom: a U-shaped (left panel) and a W-shaped (right panel) field line, given by the vector potential in Eq. (9), where $a$ is 0.5 for the U-shaped field line and -0.7 for the W-shaped field line. The grey scale map with contours (interval =1) placed at the bottom plane shows the distribution of the vertical magnetic flux at $z=0$.

where $F_{M z}$ is the energy flux across the surface and $v_{e m g}$ is given by

$$
v_{e m g} \equiv v_{z}-\frac{\mathbf{B} \cdot \mathbf{v}}{B^{2}} B_{z}
$$

At an early phase, when the boundary part of the flux tube starts to emerge ( $t=10.2$ map), a bipolar region with a PIL is formed at the surface. The horizontal field crosses the PIL from left to right. As the emergence proceeds, the vicinity of the tube axis emerges into the surface, making the horizontal field aligned with the PIL ( $t=13.8$ map).

After this axial portion emerges, the lower half of the flux tube then emerges to the surface (see the time around $t=15.4$ in the animation mentioned above). Since the field lines there have a U-shape configuration (U-loops), the emergence of U-loops causes the apparent cancellation of the vertical magnetic flux at the
PIL, called flux cancellation (Magara 2011b). During this phase, the surface distribution of magnetic field is still given by a bipolar region, while the emerging magnetic field forms a two-part structure in which the less sheared field lines overlie the inner sheared field ones. The dynamic and topological features of the two-part structure have been investigated in some detail (Magara 2006; Archontis et al. 2009).

On the other hand, the current simulation shows that a new phenomenon successively occurs; the central part of the PIL starts to rotate counterclockwise, forming a winded PIL $(t=19.2$ map). According to this rotation, the surface distribution of the magnetic field changes from a bipolar distribution to a quadrupolarlike distribution, where a new bipolar region appears inside the original bipolar region. The horizontal field connecting this new bipolar region now crosses the PIL from right to left ( $t=25$ map), which is the opposite of 


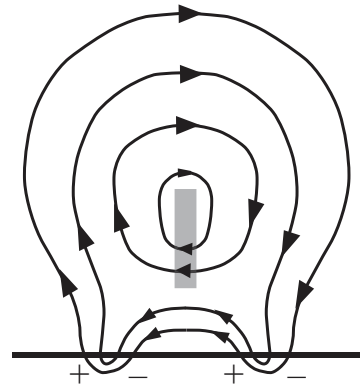

Pre-eruptive phase

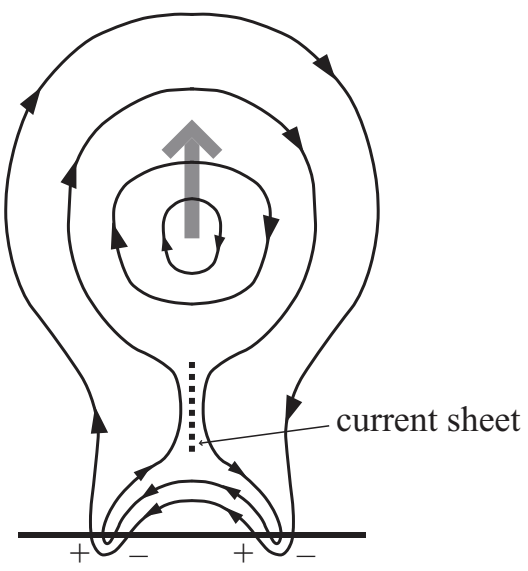

Eruptive phase

Fig. 5.- Possible field line configuration of a quadrupolar-like structure suggested by the present study. The left panel shows the pre-eruptive phase while the right one displays the eruptive phase. The shaded area in the left panel represents a filament.

what observed during the early phase $(t=10.2 \mathrm{map})$.

What causes this half-turn rotation of the PIL to form the quadrupolar-like distribution? The top panel in Fig. 4 shows the field lines giving rise to the quadrupolar-like distribution, displayed in red. It is clearly observed that the shape of these field lines changes from a simple U-shape to a $\mathrm{W}$-shape, producing a new bipolar region inside the original bipolar one. In this respect, an upflow causing net emergence plays an important role, which is continuously driven around the central part of the emerging flux tube (see the emergence velocity maps in the right panels of Fig. 3). This flow pushes the dipped part of U-loops upward, locally forming a concave-down shape. The bottom panels in Fig. 4 explain how an U-shaped and W-shaped field lines produce a bipolar and quadrupolar-like region at the surface, by using a two-dimensional model given by

$$
A_{y}(x, z)=x^{4}-\left(a x^{2}+1\right)(z-1)
$$

where $A_{y}$ represents the $y$-component of a vector potential of the magnetic field $\left(B_{x}, B_{y}, B_{z}\right)=\left(-\frac{\partial A_{y}}{\partial z}, 0, \frac{\partial A_{y}}{\partial x}\right)$ Here $a$ is a parameter taking the value $a=0.5$ for the $\mathrm{U}$-shaped field line, and $a=-0.7$ for the $\mathrm{W}$-shaped one.

\section{DISCUSSION}

The simulation used in this study suggests a possible configuration of the filament magnetic field, which is illustrated in Fig. 5. This is similar to the socalled inverse-polarity configuration shown in Fig. 1, although these two configurations are significantly different by several aspects.

The first aspect is the surface distribution of the magnetic field. The inverse-polarity configuration has a simple bipolar distribution at the surface, while a quadrupolar-like distribution is obtained from the current simulation, which is supported by observations.

Another aspect is that the inverse-polarity configuration has a neutral point below a flux rope (closed-field part in the two-dimensional case), which could develop into a current sheet where a magnetic reconnection may occur to drive an eruption of the flux rope (Shibata \& Magara 2011 and references therein). This constitutes a good explanation for the eruptive phase of a flux rope that may contain a filament inside, although it carries some difficulties in explaining why a flux rope is stable for a relatively long time before the onset of an eruption. Since in the inverse-polarity configuration a flux rope floats just above a neutral point, so if a flux rope slightly shifts upward then a vertical current sheet could develop below the flux rope, where a magnetic reconnection occurs to eject the flux rope; on the opposite, if a flux rope shifts downward, then a horizontal current sheet may develop at the neutral point to reduce the magnetic flux stored in the flux rope via magnetic reconnection. In either case, a current sheet is easy to form, and the flux rope changes a quasi-static state via reconnection, which is not suitable for the preeruptive phase during which a flux rope is quasi-static in a much longer time scale than a dynamical time scale characterized by a magnetic reconnection.

The configuration of the emerging magnetic field obtained from the present study might support a flux rope stably during the pre-eruptive phase, because there is no neutral point below the flux rope. This means that a current sheet is not easily formed, and the flux rope may keep a stable state during the pre-eruptive phase without any magnetic reconnection occurring. Of course, once a flux rope continuously rises via some destabilizing mechanism (this should be distinguished from the slight upward shift mentioned above), then 
a vertical current sheet develops below the rising flux rope to drive an eruption (see the right panel of Fig. 5). Regarding the destabilizing mechanism, the flux emergence might proceed in a multi-lobe style suggested by Magara (2007), which produces a multi-flux-rope system in the solar atmosphere. Recently, Choe et al. (2010) showed that the merging of neighboring flux ropes could lead to a grand-scale destabilization of a multi-flux-rope system. It should also be mentioned that a linear force-free modeling for the pre-eruptive phase of the filament magnetic field suggests a configuration similar to the one presented here (Aulanier \& Démoulin 1998).

In summarizing this work, we found that the subsurface upflow driven around the central part of an emerging flux tube plays an important role in causing the half-turn rotation of a PIL to form a quadrupolar-like structure in the solar atmosphere. Even if a flux tube contains an intense magnetic flux, the field strength may be reduced significantly at the tube boundary when the flux tube is twisted (Magara 2001). In this case, the flow speed will be comparable to the local Alfvén speed at the tube boundary, so that the flow can change the shape of the field lines from a U-shape to W-shape. Therefore, the condition causing the halfturn rotation of a PIL depends on the driven upflow, and on the parameters characterizing a flux tube - such as twist, field strength, tube radius, and perhaps the shape of the tube axis (Hood et al. 2009). Furthermore, if the upflow driven by the initial velocity perturbation is not so strong, then a clear half-turn rotation of a PIL observed here might not be found. An extensive study investigating relevant parameters is necessary, for a better understanding of the flux emergence and its roles in forming a magnetic structure on the Sun.

\section{ACKNOWLEDGMENTS}

This research was financially supported by the Basic Science Research Program (2010-0009258, PI: T. Magara) through the National Research Foundation of Korea (NRF) funded by the Ministry of Education, Science and Technology, as well as the World Class University (WCU) program through the NRF (R31-10016).

\section{REFERENCES}

Abbett, W. P., \& Fisher, G. H. 2003, A Coupled Model for the Emergence of Active Region Magnetic Flux into the Solar Corona, ApJ, 582, 475

Anzer, U. 1987, Modelling of the Magnetic Field of Solar Prominences, Physical Processes in Comets, Stars and Active Galaxies, 61

Archontis, V., Moreno-Insertis, F., Galsgaard, K., Hood, A., \& O'Shea, E. 2004, Emergence of Magnetic Flux from the Convection Zone into the Corona, A\&A, 426, 1047
Archontis, V., Hood, A. W., Savcheva, A., Golub, L., \& Deluca, E. 2009, On the Structure and Evolution of Complexity in Sigmoids: A Flux Emergence Model, ApJ, 691, 1276

Aulanier, G., \& Demoulin, P. 1998, 3-D Magnetic Configurations Supporting Prominences. I. The Natural Presence of Lateral Feet, A\&A, 329, 1125

Chae, J. et al. 2001, Small Magnetic Bipoles Emerging in a Filament Channel, ApJ, 548, 497

Choe, G., Lee, J. W., Cheng, C. Z., \& Kim, H. 2010, Merging and Growth of Cellular Magnetic Structures in the Solar Atmosphere Leading to a Grand Scale Eruption, 38th COSPAR Scientific Assembly, 38,1958

Gold, T., \& Hoyle, F. 1960, On the Origin of Solar Flares, MNRAS, 120, 89

Fan, Y. 2001, The Emergence of a Twisted -Tube into the Solar Atmosphere, ApJ, 554, L111

Fan, Y. 2009, Magnetic Fields in the Solar Convection Zone, Living Reviews in Solar Physics, 6, 4

Hood, A. W., Archontis, V., Galsgaard, K., \& MorenoInsertis, F. 2009, The Emergence of Toroidal Flux Tubes from Beneath the Solar Photosphere, A\&A, 503,999

Kippenhahn, R., \& Schlüter, A. 1957, Eine Theorie der Solaren Filamente. Mit 7 Textabbildungen, ZAp, 43, 36

Kuperus, M., \& Raadu, M. A. 1974, The Support of Prominences Formed in Neutral Sheets, A\&A, 31, 189

Low, B. C. 1996, Solar Activity and the Corona, Sol. Phys., 167, 217

Magara, T. 1998, Ph.D. Thesis, Kyoto University

Magara, T. 2001, Dynamics of Emerging Flux Tubes in the Sun, ApJ, 549, 608

Magara, T., \& Longcope, D. W. 2003, Injection of Magnetic Energy and Magnetic Helicity into the Solar Atmosphere by an Emerging Magnetic Flux Tube, ApJ, 586, 630

Magara, T. 2006, Dynamic and Topological Features of Photospheric and Coronal Activities Produced by Flux Emergence in the Sun, ApJ, 653, 1499

Magara, T. 2007, A Possible Structure of the Magnetic Field in Solar Filaments Obtained by Flux Emergence, PASJ, 59, L51

Magara, T. 2011a, Energy Injection Via Flux Emergence on the Sun Depending on the Geometric Shape of Magnetic Field, ApJ, 731, 122

Magara, T. 2011b, A Possible Mechanism of Flux Cancellation via U-Loop Emergence on the Sun, PASJ, 63,417

Manchester, W., IV, Gombosi, T., DeZeeuw, D., \& Fan, Y. 2004, Eruption of a Buoyantly Emerging Magnetic Flux Rope, ApJ, 610, 588 
Martin, S. F. 1998, Conditions for the Formation and Maintenance of Filaments, Sol. Phys., 182, 107

Murray, M. J., Hood, A. W., Moreno-Insertis, F., Galsgaard, K., \& Archontis, V. 2006, 3D Simulations Identifying the Effects of Varying the Twist and Field Strength of an Emerging Flux Tube, A\&A, 460, 909

Nozawa, S. 2005, Three-Dimensional Magnetohydrodynamic Simulation of Nonlinear Magnetic Buoyancy Instability of Flux Sheets with Magnetic Shear, PASJ, 57, 995

Okamoto, T. J., et al. 2008, Emergence of a Helical Flux Rope under an Active Region Prominence, ApJ, 673, L215

Parker, E. N. 1955, ApJ, The Formation of Sunspots from the Solar Toroidal Field, 121, 491

Shibata, K., \& Magara, T. 2011, Solar Flare: Magnetohydrodynamic Processes, Living Reviews in Solar Physics, in press

Tandberg-Hanssen, E. 1995, The Nature of Solar Prominences, Astrophysics and Space Science Library, 199

Vernazza, J. E., Avrett, E. H., \& Loeser, R. 1981, Structure of the Solar Chromosphere. III - Models of the EUV Brightness Components of the Quiet-Sun, ApJS, 45, 635 